\title{
Inhibition of aldehyde dehydrogenase 1 enhances the cytotoxic effect of retinaldehyde on A549 cancer cells
}

\author{
Jin Won Park ${ }^{1,2}$, Kyung-Ho Jung ${ }^{1,2}$, Jin Hee Lee ${ }^{1,2}$, Seung Hwan Moon ${ }^{1}$, Young Seok \\ Cho $^{1}$ and Kyung-Han Lee ${ }^{1,2}$ \\ ${ }^{1}$ Department of Nuclear Medicine, Samsung Medical Center, Seoul, Korea \\ ${ }^{2}$ Samsung Advanced Institute for Health Sciences \& Technology, Sungkyunkwan University School of Medicine, Seoul, Korea \\ Correspondence to: Kyung-Han Lee, email: khnm.lee@samsung.com \\ Keywords: cancer, aldehyde dehydrogenase, retinaldehyde, cancer stem cell, ROS \\ Received: January 06, $2017 \quad$ Accepted: June 26, $2017 \quad$ Published: July 25, 2017 \\ Copyright: Park et al. This is an open-access article distributed under the terms of the Creative Commons Attribution License 3.0 \\ (CC BY 3.0), which permits unrestricted use, distribution, and reproduction in any medium, provided the original author and source \\ are credited.
}

\section{ABSTRACT}

We hypothesized that aldehyde dehydrogenase1 (ALDH1) protects cancer cells from retinaldehyde-induced cytotoxicity, and that targeting this enzyme would enhance the therapeutic effect of retinaldehyde. ALDEFLUOR ${ }^{\mathrm{TM}}$ assays showed high ALDH activity in A549 and H522 cancer cells and low activity in H1666 and T47D cancer cells. Immunoblots showed that expression of ALDH1A1 and ALDH1A3 was high in A549 and H522 cells, but low in H1666 cells. HPLC confirmed that $\mathbf{N}$, N-diethylaminobenzaldehyde (DEAB) inhibits ALDH-mediated disposal of retinaldehyde in A549 cells and lysates. Treatment of A549 cells with retinaldehyde in the presence of DEAB augmented reactive oxygen species production and decreased glucose uptake and oxygen consumption. Importantly, DEAB substantially potentiated the ability of retinaldehyde to dose-dependently suppress the survival of $A 549$ and H522 cells, whereas the added effect of DEAB was minor in H1666 and T47D cells. Gene silencing with specific siRNA revealed that ALDH1A1 contributed to protection of A549 cells against retinaldehyde toxicity. These results demonstrate that ALDH1 confers protection against retinaldehyde toxicity in cancer cells.

\section{INTRODUCTION}

There is accumulating evidence that aldehyde dehydrogenase (ALDH) plays an important role in cancer [1-3]. ALDHs are a group of enzymes that catalyze the conversion of aldehydes to corresponding carboxylic acids [1]. This reaction can serve to protect cells against reactive aldehydes [4] that are potentially cytotoxic [5, 6]. Harmful effects of acetaldehyde range from increased reactive oxygen species (ROS) [7] to the formation of DNA and protein adducts [8]. Therefore, protection of cells from harmful aldehydes requires a rapid detoxification mechanism, which can be provided by their enzymatic disposal.

Among ALDHs, there is particularly great interest in ALDH1, which is widely used as a marker to identify cancer stem cells in malignancies including lung cancer
[9]. However, ALDH1 is not merely a marker of cancer stemness, but also has important roles in tumor biology [10]. ALDH1 expression is associated with poor cancer prognosis $[1,2]$ and is involved in tumor drug resistance, oxidative stress, and differentiation. Therefore, a better understanding of the pathophysiologic function of ALDH1 in cancer cells is needed. The main substrate for ALDH1 is retinaldehyde [7, 10], which is obtained from food [11]. In the cell, retinaldehyde is oxidized to retinoic acid by ALDH1. Although previously considered active only on the retina, retinaldehyde is now known to modulate the biology of a diverse range of cell types $[12,13]$.

Lung cancers frequently exhibit intrinsic and acquired resistance to anticancer agents, including recent targeted therapies [14]. It is therefore beneficial to develop new strategies to treat lung cancer. A549 cells are human adenocarcinomic alveolar basal epithelial cells that were 
developed by culturing explanted lung cancer tissue of a male patient [15]. These cells are widely used as a preclinical research model to develop new treatments for lung cancer [16] and are known to express high levels of ADLH [17], making them an appropriate cell model for our study purpose.

In this study, we used A549 cells and other cancer cells to test the hypothesis that ALDH1 protects cancer cells from the cytotoxic effects of retinaldehyde and could therefore be a potential target for cancer therapy.

\section{RESULTS}

\section{ALDH activity and major ALDH1 isoform expression}

We first evaluated the level of ALDH activity using ALDEFLUOR $^{\mathrm{TM}}$ (BODIPY-aminoacetaldehyde) assays. The results of these assays showed that $51.8 \%$ of A549 cells and $80.2 \%$ of H522 cells were ALDH positive. In comparison, only $18.4 \%$ of H1666 cells and $2.2 \%$ of T47D cells were ALDH positive (Figure 1).

Immunoblotting demonstrated strong protein bands for ALDH1A1 and ALDH1A3 in A549 cells and H522 cells. A549 cells also showed strong protein bands for ALDH3A1 (Figure 2). H1666 cells showed faint protein bands only for ALDH1A3, and none of the cells tested showed detectable ALDH1A2 expression.

\section{A high level of retinaldehyde is maintained by ALDH inhibition}

High-performance liquid chromatography (HPLC) analysis of all-trans retinaldehyde, retinoic acid, retinol, and N, N-diethylaminobenzaldehyde (DEAB) standards showed retention times of approximately $25 \mathrm{~min}, 18 \mathrm{~min}$, $19 \mathrm{~min}$, and $5 \mathrm{~min}$, respectively (Supplementary Figure 1). When retinaldehyde was incubated with A549 cell lysate for $5 \mathrm{~h}$, a very small retinaldehyde peak and a larger retinoic acid peak were observed. When we repeated the experiment in the presence of DEAB, a commonly used selective inhibitor of $\mathrm{ALDH}$, the retinaldehyde peak was greatly increased, while the retinoic acid peak became smaller (Figure 3A). We also performed HPLC of cell lysates after A549 cells had been incubated with retinaldehyde for $1 \mathrm{~h}$ in the absence or presence of DEAB. Under this condition, retinaldehyde and retinoic acid peaks appeared at somewhat earlier retention times, which was thought to represent trans-cis isomerization in living cells (Figure 3B). DEAB caused a substantial enlargement of the retinaldehyde peak, while the retinoic acid peak disappeared (Figure 3B). These findings demonstrate
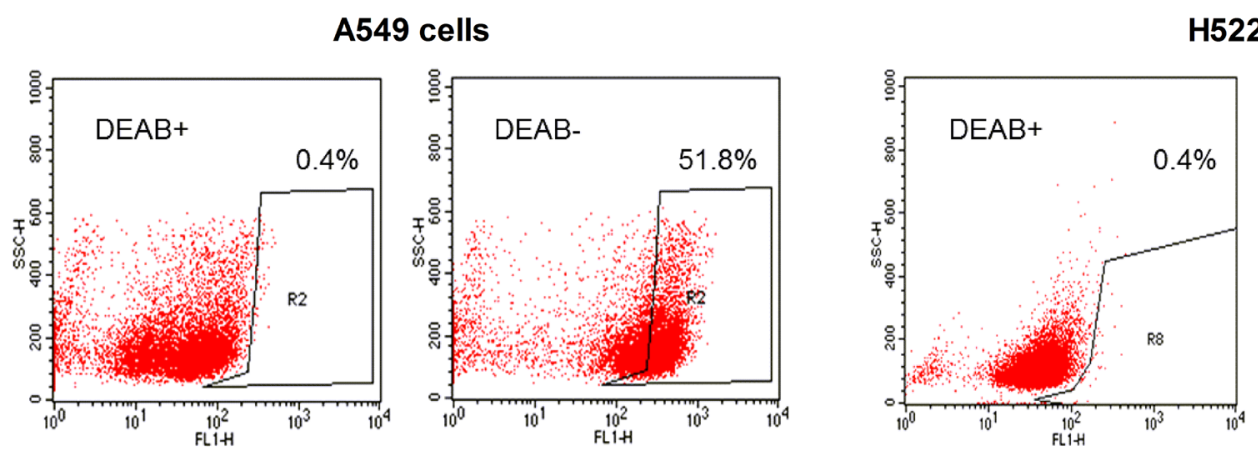

H522 cells

H1666 cells
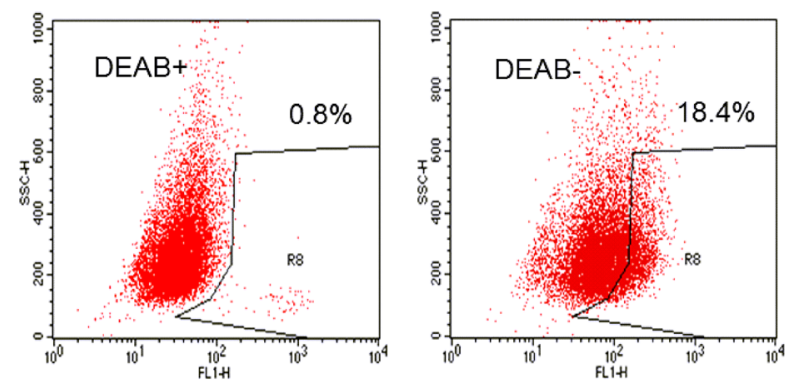

T47D cells
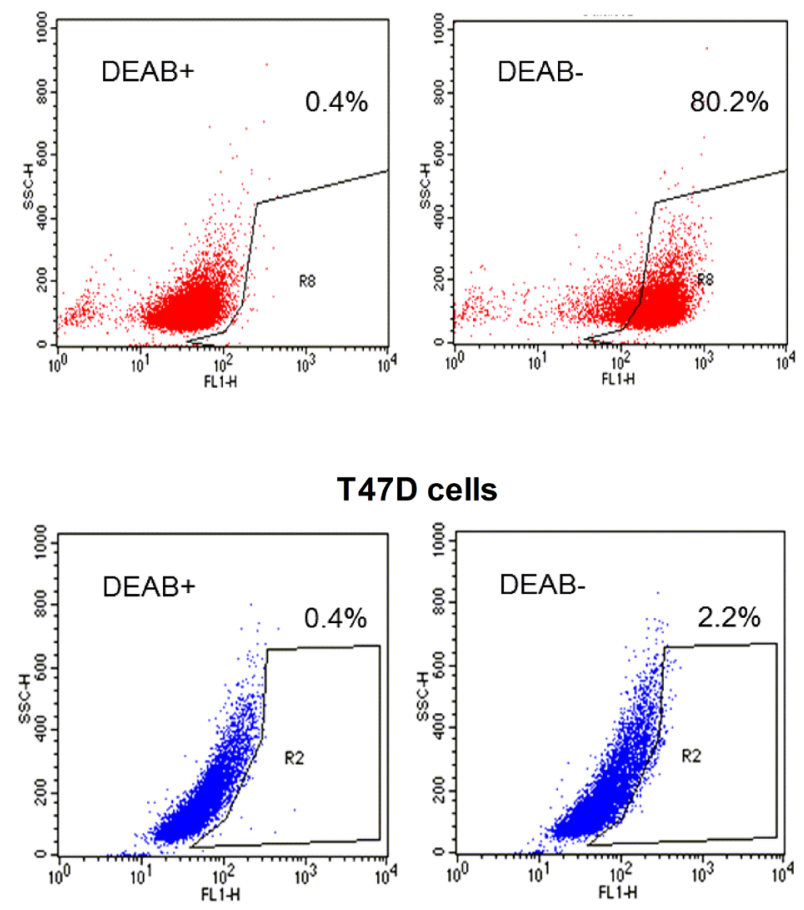

Figure 1: ALDEFLUOR ${ }^{\mathrm{TM}}$ assay measurements of ALDH activity. Representative ALDEFLUOR ${ }^{\mathrm{TM}}$ assays with FACS analysis of A549, H522, and H1666 human lung cancer cells and T47D breast cancer cells. Cells incubated with ALDEFLUOR ${ }^{\mathrm{TM}}$ reagent green dye in the presence of DEAB (left) were used to establish baseline fluorescence to define the ALDEFLUOR ${ }^{\text {TM}}$-positive area (R2). The ALDEFLUOR $^{\mathrm{TM}}$-positive population was defined as cells showing a right-shift in fluorescence following incubation with ALDEFLUOR ${ }^{\mathrm{TM}}$ in the absence of DEAB (right). 


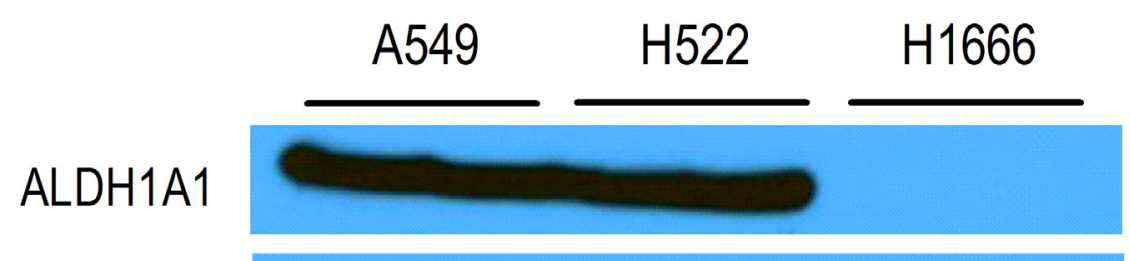

\section{ALDH1A2}

ALDH1A3

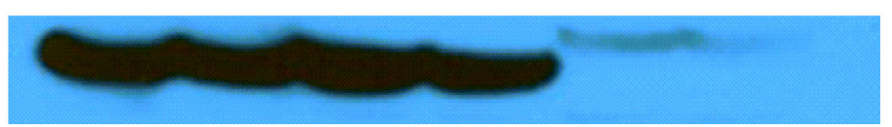

\section{ALDH3A1}

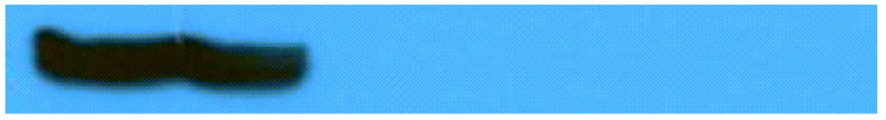

\section{$\beta$-actin}

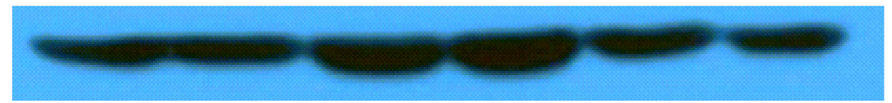

Figure 2: Immunoblots of lung cancer cell lines. Immunoblot analysis of ALDH1A1, ALDH1A2, ALDH1A3, and ALDH3A1 in A549, H522, and H1666 human lung cancer cells. $\beta$-Actin was used as a loading control.
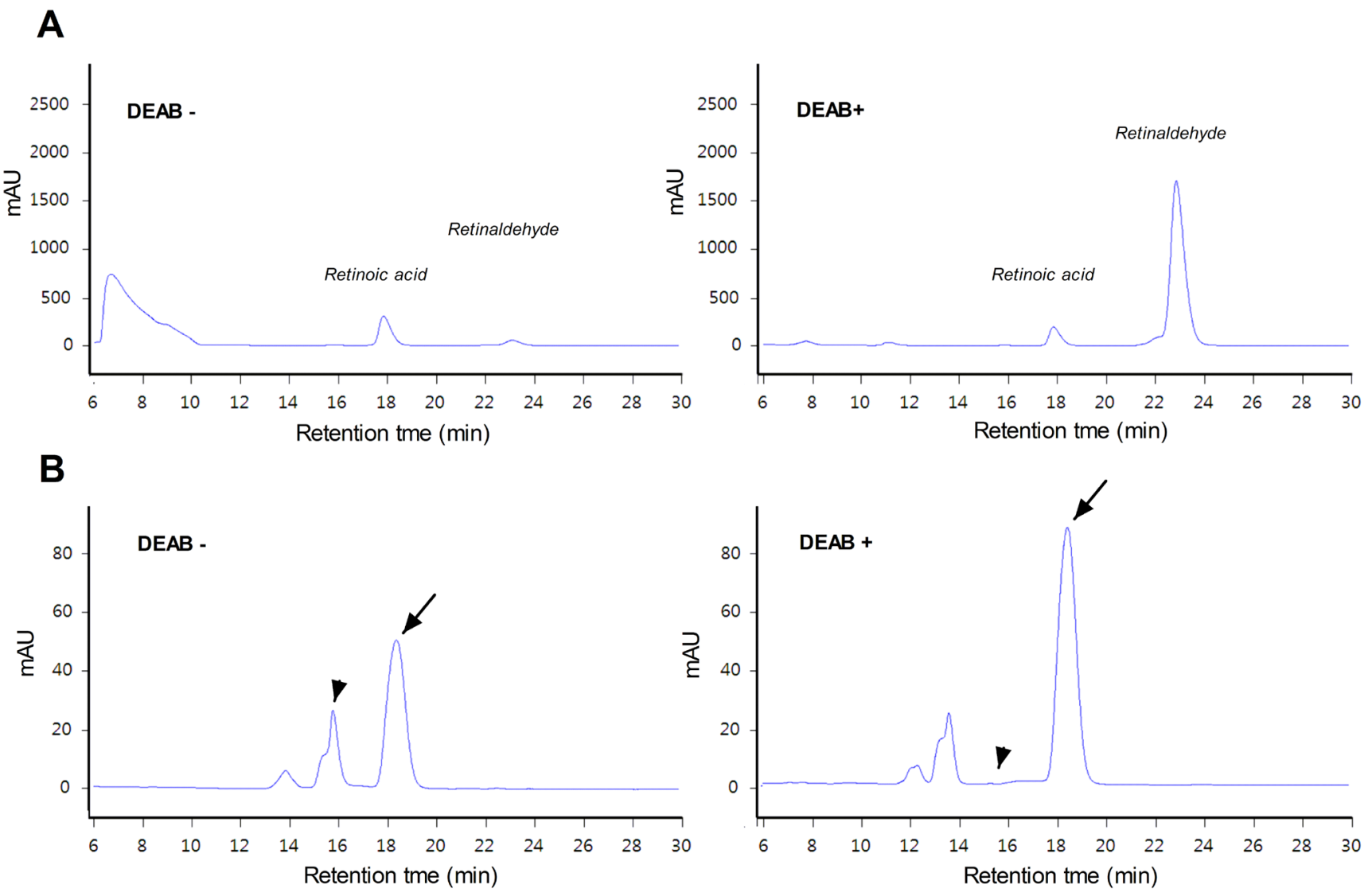

Figure 3: HPLC analysis of retinaldehyde and its metabolites. (A) HPLC chromatograms of A549 cell lysates after 5-h incubation with retinaldehyde alone or a combination of retinaldehyde and DEAB. (B) HPLC chromatograms of cytosol samples prepared from A549 cells that had been treated for $1 \mathrm{~h}$ with retinaldehyde in the presence or absence of DEAB. Arrows indicate peaks corresponding to retinaldehyde. Arrowheads indicate the retention time for the peak attributed to retinoic acid that appeared in the absence of DEAB (left) but was no longer seen when DEAB was added (right). 
that ALDH can rapidly oxidize retinaldehyde to retinoic acid, but a high level of retinaldehyde is maintained when ADLH activity is blocked by DEAB.

\section{Retinaldehyde combined with ALDH inhibition increases reactive oxygen species in A549 cells}

In initial experiments performed at a single time point of $2 \mathrm{~h}$, a combination of retinaldehyde and DEAB increased ROS production in A549 cells to $152.5 \pm 20.6 \%$ of that in controls, whereas the stimulatory effect of retinaldehyde alone did not reach statistical significance (Figure 4A). More careful examination of time-course effects showed that ROS stimulation by combined retinaldehyde and $\mathrm{DEAB}$ occurred rapidly, and that retinaldehyde alone also had a mild stimulatory effect. Levels of ROS stimulated by combined retinaldehyde and DEAB reached $141.9 \pm 16.6 \%$ of control levels by $30 \mathrm{~min}$ and further increased to $182.2 \pm 1.3 \%$ by $2 \mathrm{~h}$ (Figure $4 \mathrm{~A}$ ). Retinaldehyde alone increased ROS to $145.5 \pm 3.1 \%$ of control levels by $2 \mathrm{~h}$ (Figure 4A), which was significantly lower than that achieved in the presence of additional DEAB $(P<0.001)$.

\section{Retinaldehyde combined with DEAB suppresses glucose uptake and oxygen consumption}

When used alone, neither retinaldehyde nor DEAB significantly influenced FDG uptake of A549 cells. However, in the presence of DEAB, retinaldehyde severely reduced cellular FDG uptake to $44.4 \pm 8.7 \%$ of controls (Figure 4B).

We therefore investigated how retinaldehyde influences the oxygen consumption rate (OCR) of A549 cells. Used alone, retinaldehyde gradually caused a moderate reduction of basal OCR. The area under the curve (AUC) over $2 \mathrm{~h}$ of treatment reached $66.1 \pm 15.6 \%$ of control level. Co-treatment with retinaldehyde and DEAB decreased the $2 \mathrm{~h}$ AUC of OCR to $56.7 \pm 12.1 \%$ of controls (Figure 5). When the capacity for maximal OCR was evaluated with the proton gradient uncoupler carbonylcyanide-p-trifluoromethoxyphenylhydrazone (FCCP), combined treatment with retinaldehyde and DEAB showed a prominently greater effect. Thus, during FCCP treatment, the AUC of OCR was modestly reduced to $59.0 \pm 16.8 \%$ of controls by retinaldehyde alone, but was markedly suppressed to $25.7 \pm 10.5 \%$ of controls by addition of DEAB (Figure 5).

\section{Potentiation of retinaldehyde cytotoxicity by DEAB}

Sulforhodamine-B (SRB) assays showed that a high concentration of retinaldehyde alone decreased viability of all cancer cells tested, but with different magnitudes. When treated with $50 \mu \mathrm{M}$ retinaldehyde, cell viability was lowest for H1666 cells $(1.1 \pm 1.4 \% ; P<0.001$ compared with all other cell types), followed by T47D cells $(23.7$ $\pm 8.3 \%)$ and A549 cells $(35.4 \pm 5.4 \%)$, and was highest for $\mathrm{H} 522$ cells $(80.7 \pm 5.1 \% ; P<0.001$ compared with all other cell types; Figure 6A). The potentiating effect of combined treatment with $\mathrm{DEAB}$ on retinaldehyde cytotoxicity varied according to cell type. Although DEAB did not further reduce the viability of T47D cells, it significantly reduced viability of other cell types and had a particularly marked effect on H522 cells. Thus, in the presence of DEAB, $20 \mu \mathrm{M}$ retinaldehyde reduced A549 cell viability from $80.2 \pm 2.4 \%$ to $46.8 \pm 5.4 \%$, H1666 cell viability from $90.8 \pm 5.6 \%$ to $66.8 \pm 3.1 \%$, and $\mathrm{H} 522$ cell viability from $103.6 \pm 2.0 \%$ to $33.7 \pm 2.4 \%$ (all $P<0.001$;
A

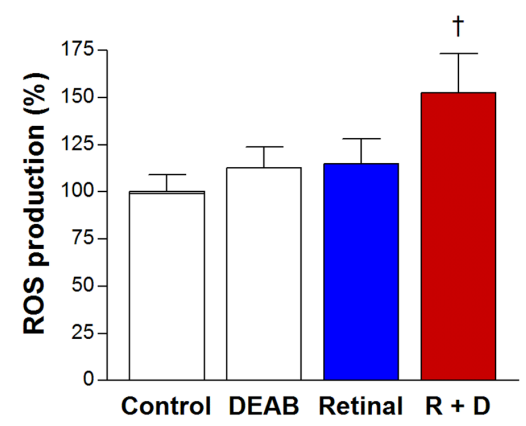

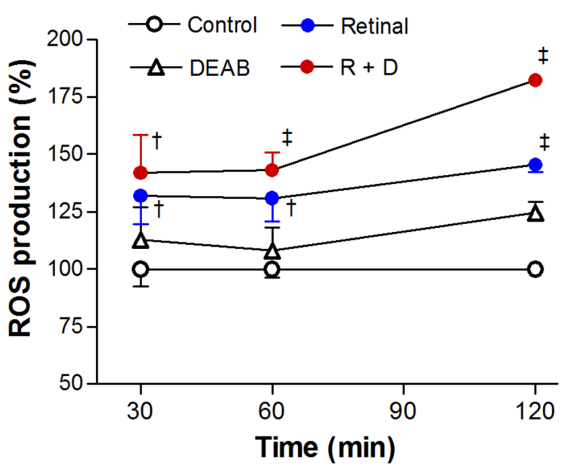

B

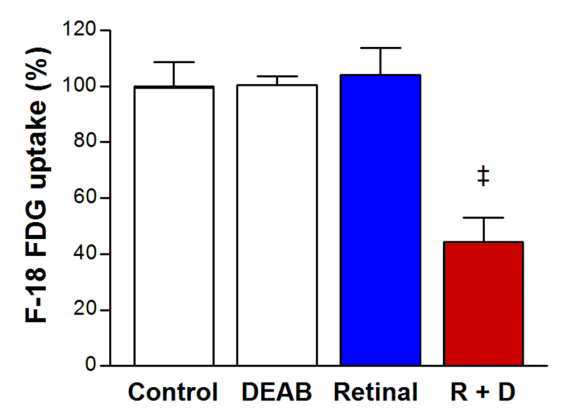

Figure 4: Retinaldehyde with ALDH inhibition stimulates ROS production and reduces FDG uptake. (A) Intracellular ROS was measured by CMH2DCFDA fluorescence in A549 cells treated for $2 \mathrm{~h}$ with $30 \mu \mathrm{M}$ retinaldehyde and/or $100 \mu \mathrm{M}$ DEAB (left). The temporal course of ROS production from $30 \mathrm{~min}$ to $2 \mathrm{~h}$ of treatment as above (right). (B) FDG uptake of A549 cells after $2 \mathrm{~h}$ treatment as above. All data are mean $\pm \mathrm{SD}$ of percentage values relative to untreated controls $(\mathrm{n}=5$ for $\mathrm{A}$, and $\mathrm{n}=4$ for $\mathrm{B})$ obtained from a single representative experiment. $\uparrow P<0.005, \ddagger P<0.001$, compared with controls treated with DMSO vehicle. 
Figure 6A). The magnitude of reduction in viability was significantly greater for H522 cells compared with all other cell types $(P<0.001)$.

Graded doses of DEAB showed only weak effects on A549 and T47D cell viability (Figure 6B). When 30 $\mu \mathrm{M}$ of retinaldehyde was added, A549 cell viability decreased in a DEAB concentration-dependent manner, reaching $15.6 \pm 10.5 \%$ of control levels with $200 \mu \mathrm{M}$ DEAB (Figure 6B). In contrast, T47D cell viability in response to graded doses of $\mathrm{DEAB}$ was not influenced by the presence of $30 \mu \mathrm{M}$ retinaldehyde (Figure $6 \mathrm{~B}$ ).

\section{Gene silencing shows that ALDH1A1 contributes to protection against retinaldehyde}

At 2 days following transfection with ALDH1A1specific siRNA, A549 cell viability was $57.0 \pm 2.2 \%$ of that of control cells transfected with scrambled siRNA (Figure 7A). This was thought to represent the slower proliferation of cells with ALDH1A1 silencing. In cells transfected with control siRNA, treatment with $50 \mu \mathrm{M}$ retinaldehyde reduced cell viability to $52.6 \pm 3.3 \%$ of untreated cells. In cells transfected with ALDH1A1 siRNA, $50 \mu \mathrm{M}$ retinaldehyde caused a significantly greater reduction in viability, which reached $38.2 \pm 1.1 \%$ of that of untreated cells (Figure 7A).

\section{Retinaldehyde combined with DEAB increases apoptotic cell death}

Cell death enzyme-linked immunosorbent assay (ELISA) showed that apoptosis in A549 cells treated with retinaldehyde alone increased to $172.5 \pm 26.2 \%$ of that in untreated control cells. Treatment with a combination of
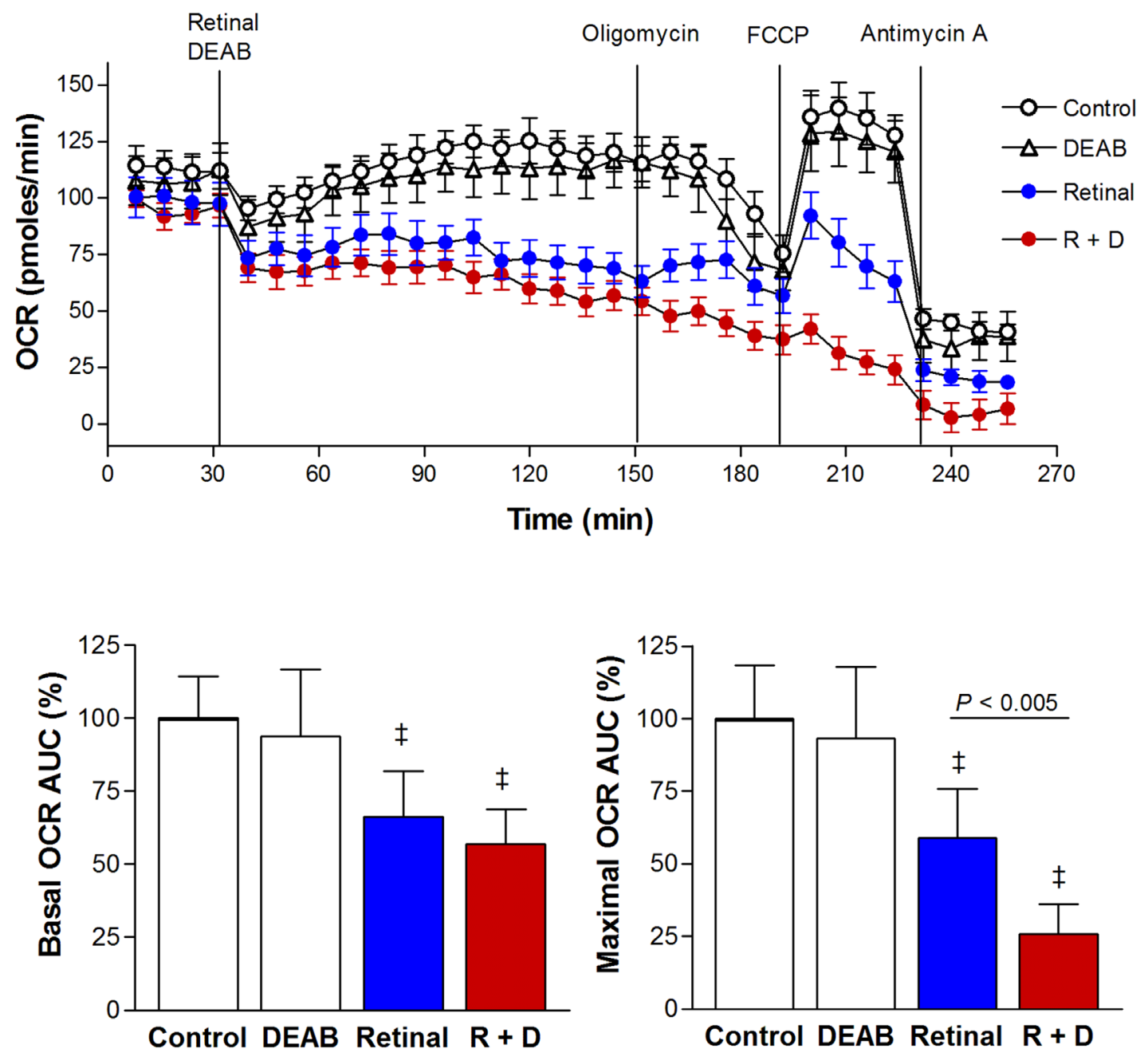

Figure 5: ALDH inhibition potentiates the ability of retinaldehyde to suppress cellular OCR. OCR of A549 cells treated as above at baseline (30 to $150 \mathrm{~min}$ ) and following sequential addition of oligomycin, FCCP, and antimycin A (top). AUC of the measured OCR of each group of cells at baseline (bottom, left) and during FCCP stimulation (bottom, right). Data are mean \pm SD of percentage values relative to untreated controls obtained from a single representative experiment with 5 samples per group. $\ddagger P<0.001$, compared with controls. 
A

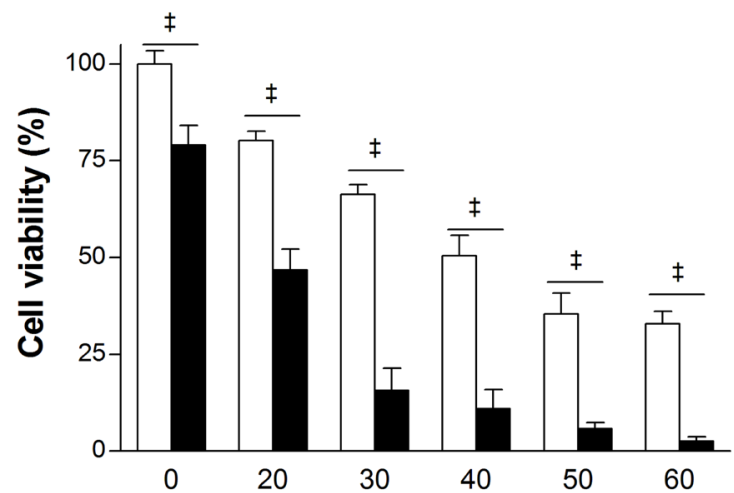

Retinaldehyde concentration ( $\mu \mathrm{M})$

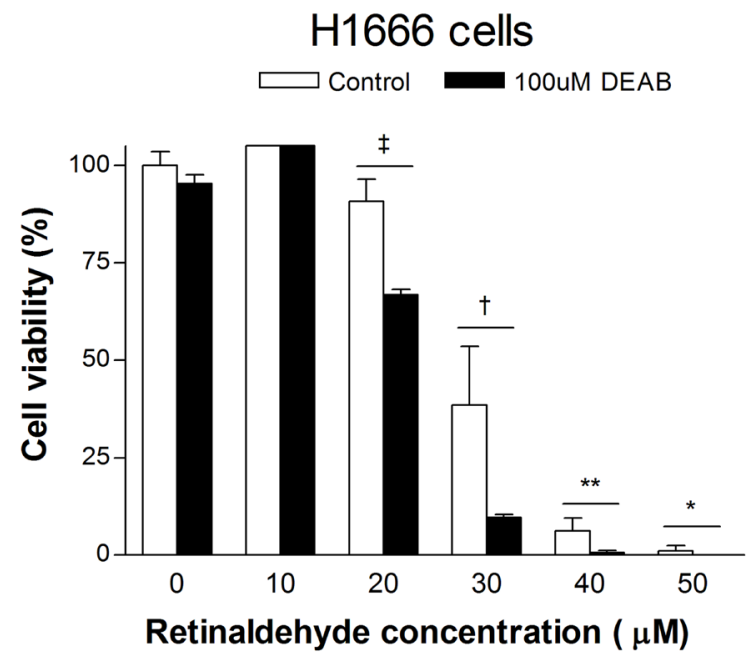

B

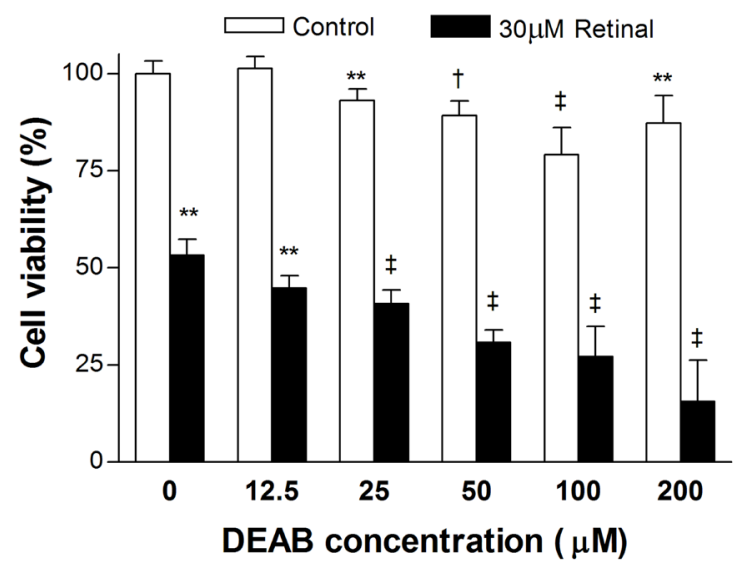

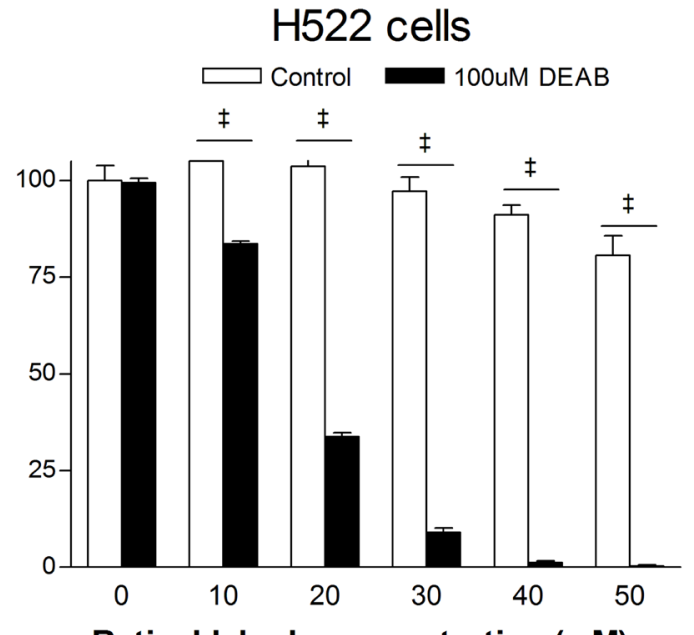

Retinaldehyde concentration ( $\mu \mathrm{M})$
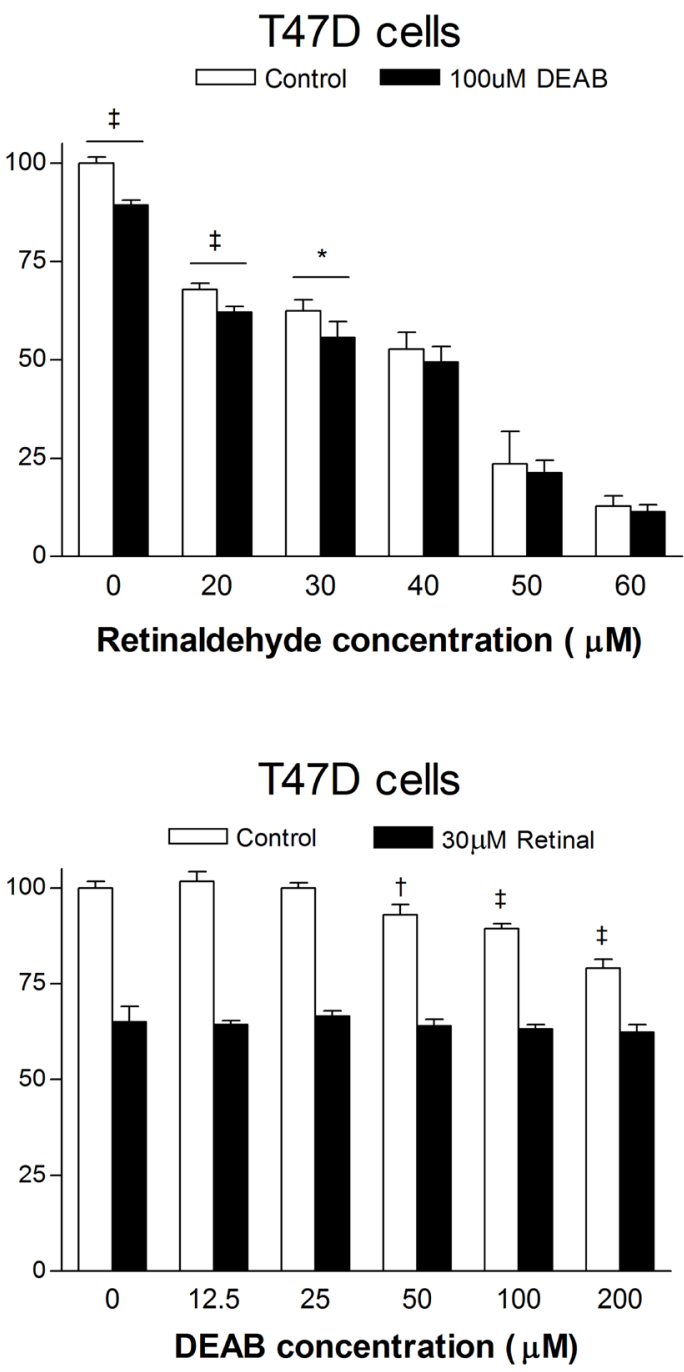

Figure 6: Effects of retinaldehyde and ALDH inhibition on cell viability. (A) SRB assays of A549, H522, H1666, and T74D cell survival after $24 \mathrm{~h}$ treatment with graded doses of retinaldehyde with or without $100 \mu \mathrm{M}$ DEAB. (B) SRB assays of A549 and T74D cell survival after $24 \mathrm{~h}$ treatment with graded doses of DEAB with or without $30 \mu \mathrm{M}$ retinaldehyde. Bars are mean $\pm \mathrm{SD}$ of percentage values relative to untreated controls $(\mathrm{n}=5)$ from a single representative experiment. ${ }^{*} P<0.05,{ }^{* *} P<0.01,{ }^{\dagger} P<0.005,{ }^{\ddagger} P<0.001$, compared with untreated controls. 
retinaldehyde and $\mathrm{DEAB}$ resulted in a significantly greater rate of apoptosis that reached $242.1 \pm 35.0 \%$ of control levels (Figure 7B).

\section{DISCUSSION}

Our study demonstrates that retinaldehyde efficiently and dose-dependently suppresses cancer cell survival. In A549 cancer cells, retinaldehyde toxicity was substantially potentiated by a specific ALDH inhibitor. This enhanced cytotoxic effect was accompanied by stimulation of ROS production, impairment of mitochondrial respiration, and increased apoptotic cell death.
In our study, we used A549 human cancer cells because they are widely used as an in vitro model to develop new lung cancer treatments and are known to have high ALDH activity [17]. In comparison, T47D human breast cancer cells have low ALDH activity [2]. We also used additional human adenocarcinoma cell lines, H522 cells and H1666 cells, which we found to have high and low ALDH activity, respectively. ALDEFLUOR ${ }^{\mathrm{TM}}$ assay results are largely determined by ALDH1 activity (and particularly ALDH1A1 activity), although ALDH3A1 and ALDH7A1 can also contribute [10]. In addition to ALDEFLUOR ${ }^{\mathrm{TM}}$ assays, we also performed immunoblots and found that A549 cells and H522 cells have strong
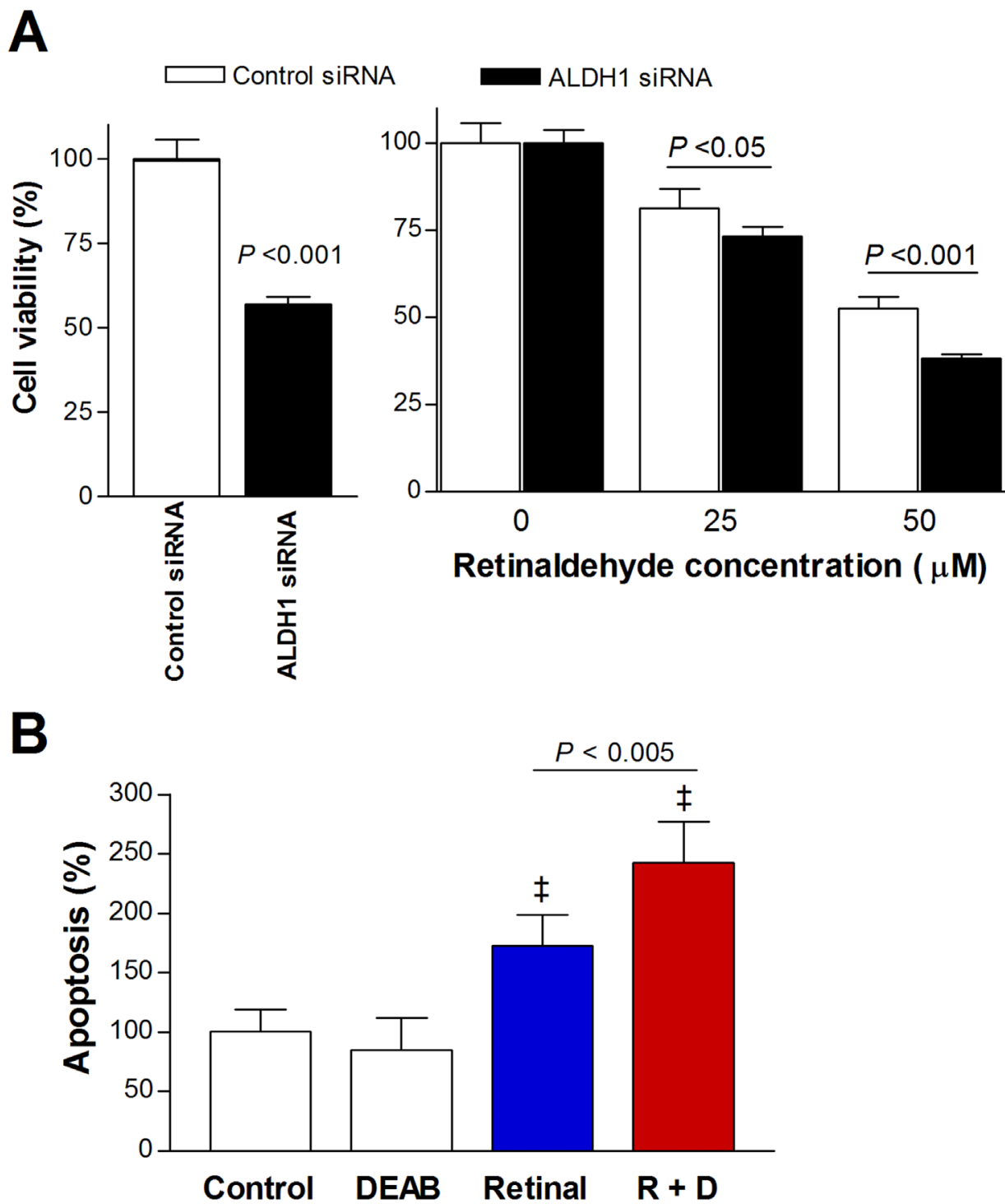

Figure 7: Retinaldehyde cytotoxicity under ALDH1A1 silencing and apoptosis induced by retinaldehyde. (A) SRB assays of A549 cells $48 \mathrm{~h}$ after transfection with ALDH1A1-specific and scrambled control siRNA (left). Relative viability of transfected A549 cells is shown after 24-h treatment with 25 and $50 \mu \mathrm{M}$ retinaldehyde (right). (B) Immunosorbent assays of cell death enzymes showing influence of 15 -h treatment with retinaldehyde and/or DEAB on A549 cell apoptosis. All bars are mean \pm SD of percentage values relative to controls obtained from a single representative experiment $(\mathrm{n}=4, \mathrm{~A} ; \mathrm{n}=6, \mathrm{~B})$. $\$ P<0.001$, compared with untreated control cells. 
ALDH1A1 and ALDH1A3 expression but no detectable ALDH1A2 expression. H1666 cells had weak ALDH1A3 expression with undetectable expression of ALDH1A1. Our results for A549 cells are consistent with a previous study showing ALDH1A1 and ALDH3A1 expression by these cells [17].

Retinaldehyde is rapidly metabolized either by oxidation to retinoic acid via ALDHs or by reduction to retinol via retinol dehydrogenases [18]. In a previous study, retinaldehyde was rapidly metabolized by retinal epithelial cells and became undetectable by $4.5 \mathrm{~h}$, leaving retinol, retinoic acid, and retinyl esters [19]. Under our HPLC conditions, all-trans retinol and retinoic acid were identified as distinct peaks that eluted earlier than the retinaldehyde peak. When retinaldehyde was applied to A549 cell lysate or to live A549 cells, there was a rapid decline in retinaldehyde concentration with an increase in its metabolites. However, a high retinaldehyde concentration was maintained in the presence of DEAB. This demonstrates that blocking ALDH activity delays enzymatic removal of retinaldehyde in cells. A shift in the retention times of retinaldehyde and retinoic acid after incubation with cells might represent trans-cis isomerization of retinoids. All-trans retinaldehyde can be converted into isoforms with different HPLC retention times [20], and cis isoform isomerization of all-trans retinaldehyde has been reported in retinal epithelial cells [21].

Reactive aldehydes can increase ROS production. This is exemplified by the reported ability of acetaldehyde to stimulate ROS in hepatic stellate cells $[22,23]$ and neuroblastoma cells [24]. Retinaldehyde has been shown to similarly stimulate ROS generation in retinal epithelial cells [25], and this response has been proposed to contribute to retinal macular degeneration [26]. A recent study found that patient tissue-derived ovarian cancer cells with strong ALDH activity had lower ROS levels [27]. In our study, ROS levels were increased when A549 cells were treated with retinaldehyde in the presence of DEAB. Taken together, these findings are consistent with the notion that ALDH mitigates cellular oxidative stress by scavenging aldehydes [7].

When we evaluated mitochondrial function, glucose uptake by A549 cells was unaffected by retinaldehyde or DEAB alone, but was significantly suppressed by a combination of retinaldehyde and DEAB. Also, oxygen consumption rate was moderately decreased by retinaldehyde alone and appeared to be suppressed to a slightly greater extent when DEAB was present. Furthermore, a profound difference was observed for FCCP-stimulated OCR, which represents maximal mitochondrial respiratory capacity. Cells treated with a combination of retinaldehyde and DEAB displayed a nearly complete flat response to FCCP, whereas the response was only modestly reduced by retinaldehyde alone. These findings demonstrate that cancer cell mitochondrial function is suppressed by retinaldehyde in the absence of ALDH activity.

A suppressive effect of retinaldehyde on cell survival has been previously demonstrated in retinal epithelial cells [25]. When we examined cancer cell viability by SRB assays, retinaldehyde alone dosedependently decreased the viability of all cancer cells tested but with different potencies. The cytotoxic effect was greatest for H1666 cells, followed by T47D cells and A549 cells, whereas it was minimal for H522 cells. Conversely, DEAB-induced susceptibility to retinaldehyde was greatest for H522 cells and weakest for H1666 cells and T47D cells. These findings suggest that ALDH offers protection against retinaldehyde toxicity in a manner that is effectively blocked by DEAB.

A potential association between cytotoxicity of retinoids and the activity of enzymes involved in their metabolism has previously been pointed out. In kidney HEK293 cells, retinaldehyde toxicity was decreased by expression of retinol dehydrogenase [28]. In Sertoli cells, ALDH inhibition potentiated retinol-induced ROS production and cell damage [29]. In another study, forced expression of retinol dehydrogenase in MCF7 breast cancer cells lacking ALDH resulted in increased cytotoxicity of retinol that was attributed to the formation of retinaldehyde [30].

DEAB is widely used in ALDEFLUOR ${ }^{\mathrm{TM}}$ assays to identify stem cells that express ALDH1. Although generally considered a selective ALDH1A1 inhibitor, DEAB is also able to block ALDH2, an isoform responsible for alcohol-induced acetaldehyde detoxification [31]. Furthermore, DEAB has also been reported to inhibit other ALDH1 isoforms including ALDH1A2, ALDH1A3, and ALDH1B1 [32]. Therefore, the ability of DEAB to potentiate retinaldehyde toxicity cannot be attributed to blockage of the activity of a specific ALDH isoform.

Using gene silencing experiments, we confirmed that retinaldehyde cytotoxicity was significantly potentiated when A549 cells were transfected with siRNA specific for ALDH1A1 compared with scrambled siRNA. Our focus on silencing ALDH1A1 was motivated not only because it was abundantly expressed in A549 cells, but also because of its important roles in cancer biology. ALDH1A1 is one of three highly conserved cytosolic isozymes that catalyze the oxidation of retinaldehyde to retinoic acid with high affinity. In cancer, this isoform has an important role in modulating cell proliferation and differentiation. ALDH1A1 overexpression, which is observed in various cancer types, has been associated with cancer cell stemness, treatment resistance, and poor patient prognosis [1]. Our results indicate that ALDH1A1 contributes to a protective effect against retinaldehyde toxicity that is blocked by DEAB. Nevertheless, it should be mentioned that we did not evaluate the effect of silencing other ALDH1 isoforms, and it is therefore 
possible that ALDH1A3 also contributes to protection against retinaldehyde cytotoxicity.

Finally, cell death ELISA demonstrated that the cytotoxic effect of retinaldehyde was accompanied by increased apoptosis. A previous study showed that retinaldehyde induced retinal epithelial cell death by triggering apoptosis, and that this was attributed to ROS-induced DNA damage and p53 activation [25]. Furthermore, SH-SY5Y neuroblastoma cancer cells treated with acetaldehyde showed cytotoxic effects via apoptotic signaling and inhibition of cell survival pathways [24]. Our results suggest that retinaldehyde cytotoxicity in cancer cells with blocked ALDH activity might also occur through promotion of apoptosis.

Our findings support the possibility that combining retinaldehyde with drugs that inhibit ALDH1A1 might offer potential benefit for cancer treatment. Retinaldehyde is normally present in living bodies, and cancer stem cells use significant amounts of retinaldehyde for differentiation $[1,2]$. This suggests that ALDH inhibitors might be useful for cancer treatment without the need for additional retinaldehyde. It should be pointed out, however, that DEAB has limited specificity for ALDH isoforms, and newer ALDH inhibitors with greater specificity might be required for this purpose.

In conclusion, our results confirm that retinaldehyde exerts dose-dependent cytotoxicity on cancer cells, and that this effect is potentiated by blocking ALDH1 activity. These data indicate that an important role of ALDH1 in cancer cells is to confer protection by detoxifying retinaldehyde through enzymatic disposal, which could offer a potential therapeutic target.

\section{MATERIALS AND METHODS}

\section{Cell culture}

A549, H522, and H1666 human lung adenocarcinoma cells and T47D human breast cancer cells were from the Korea Cell Line Bank (Republic of Korea, Seoul) and the American Type Culture Collection (Manassas, VA). Cells were maintained in RPMI 1640 media (Lonza, Switzerland) supplemented with 10\% fetal bovine serum (Serena, Germany) and 1\% penicillin/ streptomycin (Lonza) at $37^{\circ} \mathrm{C}$ and $5 \% \mathrm{CO}_{2}$ in a humidified atmosphere. Media were replaced with phenol red-free RPMI 1640 (Gibco, MA) with 10\% fetal bovine serum 48 $\mathrm{h}$ before experiments.

\section{ALDH activity assay}

ALDH activity was measured using an ALDEFLUORTM assay kit (Stemcell Tech, BC, Canada) according to the manufacturer's manual. Cells were resuspended in ALDEFLUOR ${ }^{\mathrm{TM}}$ assay buffer containing $1 \mu \mathrm{M}$ ALDEFLUOR ${ }^{\mathrm{TM}}$ dye. ALDH activity was blocked with $15 \mu \mathrm{M}$ of DEAB, a commonly used selective inhibitor of ALDH isoenzymes in cancer and stem cells. After incubation at $37^{\circ} \mathrm{C}$ and $5 \% \mathrm{CO}_{2}$ for $30 \mathrm{~min}$ in a humidified atmosphere, cells were washed with ALDEFLUORTM assay buffer. Finally, 10,000 cells per sample were analyzed using a FACS Calibur flow cytometer with CellQuest software (Becton-Dickinson, NJ).

\section{Immunoblotting}

Cells were washed with PBS and solubilized in PRO-PREPTM protein extraction solution (iNtRON biotechnology, Korea) for $15 \mathrm{~min}$ at $4^{\circ} \mathrm{C}$. Cell debris was eliminated by centrifugation at $14,000 \mathrm{rpm}$ for 10 $\min$ at $4^{\circ} \mathrm{C}$. The supernatant was analyzed for protein content by the Bradford method, and $20 \mu \mathrm{g}$ of protein was separated on a $10 \%$ polyacrylamide gel. The protein was transferred to a hydrobond ECL nitrocellulose membrane (Amersham Biosciences; Piscataway, NJ) and incubated overnight at $4^{\circ} \mathrm{C}$ with polyclonal antibody against ALDH1A1 (Abcam; Cambridge, MA; 1:1000 dilution), ALDH1A2 (Abcam; Cambridge, MA; 1:1000 dilution), ALDH1A3 (Genetex; Cambridge, MA; 1:1000 dilution), or ALDH3A1 (Cusabio; CA; 1:1000 dilution) in Trisbuffered saline (50 mM Tris, $\mathrm{pH} 7.5,150 \mathrm{mM} \mathrm{NaCl}$ ) with $0.05 \%$ polysorbate- 20 containing $5 \%$ skim milk. After washing three times for $10 \mathrm{~min}$ with Tris-buffered saline with Tween 20, the membrane was incubated with secondary antibodies for $1 \mathrm{~h}$ at room temperature. Immune reactive protein was detected with an enhanced chemiluminescence kit and quantified using Quantity One software (Bio-Rad Laboratories, CA).

\section{HPLC analysis}

HPLC analysis was performed with two types of sample. The first was untreated A549 cell lysates that were reacted with $100 \mu \mathrm{g}$ retinaldehyde with or without $100 \mu \mathrm{g}$ DEAB at $37^{\circ} \mathrm{C}$ for $5 \mathrm{~h}$. HPLC was performed on a Spectra system P4000 (Thermo, MA) equipped with a reverse-phase $\mathrm{C} 18$ column $\left(4.6^{*} 250 \mathrm{~mm}\right)$ (YMC, Japan) and a UV2000 detector. Elution was performed with $80 \%$ acetonitrile in water for $30 \mathrm{~min}$ at a flow rate of $1 \mathrm{ml} / \mathrm{min}$. Samples were analyzed at specific absorption wavelength of $298 \mathrm{~nm}$.

The second type of sample was obtained from A549 cells that had been treated with $30 \mu \mathrm{M}$ retinaldehyde with or without $100 \mu \mathrm{M}$ DEAB for $1 \mathrm{~h}$. Cells were washed with phosphate-buffered saline and lysed by pumping through a 23-G needle in $100 \mu \mathrm{l}$ distilled water. After centrifugation at $14,000 \mathrm{rpm}$ for $10 \mathrm{~min}$, the supernatants were subjected to HPLC analysis.

\section{ROS production}

Cells were cultured in 96-well black plates. Six hours before measurement, the culture medium was 
replaced with phenol red-free RPMI 1640 containing 10\% fetal bovine serum and $10 \mu \mathrm{M}$ CMH2DCFDA (Molecular Probes, Invitrogen, CA). Fluorescence was measured on a microplate reader using $490 \mathrm{~nm}$ excitation and 510 to 570 $\mathrm{nm}$ emission wavelengths.

\section{FDG uptake}

Cells were incubated with $175-370 \mathrm{kBq}$ FDG for $40 \mathrm{~min}$ at $37^{\circ} \mathrm{C}$ and then rapidly washed twice with cold phosphate-buffered saline, lysed with $0.01 \mathrm{~N} \mathrm{NaOH}$, and measured for cell-associated radioactivity on a highenergy $\gamma$ counter (PerkinElmer, MA).

\section{OCR}

Cells were seeded into Seahorse XF24 24-well plates (Seahorse Bioscience, MA) at 20,000 cells per well and equilibrated with $525 \mu$ phenol red-free RPMI 1640 ( $\mathrm{pH} \mathrm{7.4)} \mathrm{without} \mathrm{sodium} \mathrm{bicarbonate} \mathrm{or} \mathrm{fetal} \mathrm{bovine}$ serum at $37^{\circ} \mathrm{C}$ without $\mathrm{CO}_{2}$ for $1 \mathrm{~h}$ before the assay. Oxygen concentration of the media was measured on a Seahorse XF24 extracellular flux analyzer (Seahorse Bioscience, MA) with solid state sensor probes. OCR was measured during basal respiration and after treatment with retinaldehyde with or without DEAB. Oligomycin (4 $\mu \mathrm{M}$, Sigma) was used to inhibit complex V, FCCP (10 $\mu \mathrm{M}$, Sigma) was used to uncouple the proton gradient, and antimycin A (10 $\mu \mathrm{M}$, Sigma) was used to inhibit complex III. These agents were sequentially added to the media of cells, while OCR was automatically calculated, recorded, and plotted using Seahorse XF24 software version 1.8. Mean OCR over certain time points was quantified as the AUC.

\section{ALDH1A1 knockdown by siRNA}

Cells were transfected with ALDH1A1 siRNA (Santa Cruz Biotechnology, TX) or a negative control siRNA (Cell Signaling Technology, MA) in Opti-MEM with lipofectamine RNAi-MAX (Invitrogen, CA) according to the manufacturer's manual. For transfection, $200 \mathrm{nM}$ of siRNA was diluted in $25 \mu \mathrm{l}$ Opti-MEM transfection medium (solution A). Solution B was prepared by adding $1.5 \mu \mathrm{l}$ RNAimax transfection reagent to $25 \mu \mathrm{l}$ Opti-MEM transfection medium. Solutions A and B were mixed and incubated for $5 \mathrm{~min}$ at room temperature. This mixture was added to 96 -well plates and incubated in a $\mathrm{CO}_{2}$ incubator at $37^{\circ} \mathrm{C}$. Cells were further maintained for $48 \mathrm{~h}$ before drug treatment.

\section{Cell viability assay}

Cells were fixed with $10 \%(\mathrm{w} / \mathrm{v})$ trichloroacetic acid and stained with SRB for $30 \mathrm{~min}$. Excess dye was removed by repeated washing with $1 \%(\mathrm{v} / \mathrm{v})$ acetic acid, and protein-bound dye was dissolved in $10 \mathrm{mM}$ Tris base solution for determination of optical density at $510 \mathrm{~nm}$ on a micro-plate reader.

\section{Apoptosis assay}

Apoptosis was evaluated using a cell death detection ELISA plus kit (Roche, Switzerland). Briefly, after cell treatment for $15 \mathrm{~h}$, culture media was replaced with fresh media, and cells were harvested with lysis buffer $1 \mathrm{~h}$ later. Cell lysates were incubated in streptavidin-coated plates with $80 \mu \mathrm{l}$ of a mixture anti-histone-biotin and anti-DNA-POD with shaking at RT for $2 \mathrm{~h}$. After washing with incubation buffer, the plates were reacted with 2,2'-azinobis (3-ethylbenzothiazoline-6-sulfonic acid)diammonium salt (ABTS) solution 10-20 min until the color of the buffer changed. Samples were finally analyzed at $405 \mathrm{~nm}$ on a micro-plate reader.

\section{Data analysis}

Data are expressed as the mean \pm SD. Significance of difference was analyzed by two-tailed unpaired Student's t-tests for two groups and by ANOVA with Tukey post-hoc tests for three or more groups. $P$ values $<0.05$ were considered statistically significant.

\section{ACKNOWLEDGMENTS}

This research was supported by the Basic Science Research Program through the National Research Foundation of Korea (NRF) funded by the Ministry of Science, ICT, and Future Planning (NRF2015R1A2A2A01006419).

\section{CONFLICTS OF INTEREST}

The authors declare no conflicts of interest.

\section{REFERENCES}

1. Tomita H, Tanaka K, Tanaka T, Hara A. Aldehyde dehydrogenase 1A1 in stem cells and cancer. Oncotarget. 2016; 7:11018-11032. https://doi.org/10.18632/ oncotarget.6920.

2. Deng S, Yang X, Lassus H, Liang S, Kaur S, Ye Q, Li C, Wang LP, Roby KF, Orsulic S, Connolly DC, Zhang Y, Montone K, et al. Distinct expression levels and patterns of stem cell marker, aldehyde dehydrogenase isoform 1 (ALDH1), in human epithelial cancers. PLoS One. 2010; 5:e10277.

3. Januchowski R, Wojtowicz K, Zabel M. The role of aldehyde dehydrogenase (ALDH) in cancer drug resistance. Biomed Pharmacother. 2013; 67:669-680.

4. Marchitti SA, Brocker C, Stagos D, Vasiliou V. Non-P450 aldehyde oxidizing enzymes: the aldehyde dehydrogenase 
superfamily. Expert Opin Drug Metab Toxicol. 2008; 4:697-720.

5. Brooks PJ, Theruvathu JA. DNA adducts from acetaldehyde: implications for alcohol-related carcinogenesis. Alcohol. 2005; 35:187-193.

6. Jacobs AT, Marnett LJ. Systems analysis of protein modification and cellular responses induced by electrophile stress. Acc Chem Res. 2010; 43:673-683.

7. Singh S, Brocker C, Koppaka V, Chen Y, Jackson BC, Matsumoto A, Thompson DC, Vasiliou V. Aldehyde dehydrogenases in cellular responses to oxidative/ electrophilic stress. Free Radic Biol Med. 2013; 56:89-101.

8. Yu HS, Oyama T, Isse T, Kitagawa K, Pham TT, Tanaka M, Kawamoto T. Formation of acetaldehyde-derived DNA adducts due to alcohol exposure. Chem Biol Interact. 2010; 188:367-375.

9. Jiang F, Qiu Q, Khanna A, Todd NW, Deepak J, Xing L, Wang H, Liu Z, Su Y, Stass SA, Katz RL. Aldehyde dehydrogenase 1 is a tumor stem cell-associated marker in lung cancer. Mol Cancer Res. 2009; 7:330-338.

10. Marcato P, Dean CA, Giacomantonio CA, Lee PW. Aldehyde dehydrogenase: its role as a cancer stem cell marker comes down to the specific isoform. Cell Cycle. $2011 ; 10: 1378-1384$

11. D'Ambrosio DN, Clugston RD, Blaner WS. Vitamin A metabolism: an update. Nutrients. 2011; 3:63-103.

12. Desvergne B. Retinaldehyde: more than meets the eye. Nat Med. 2007; 13:671-673.

13. Ziouzenkova O, Orasanu G, Sharlach M, Akiyama TE, Berger JP, Viereck J, Hamilton JA, Tang G, Dolnikowski GG, Vogel S, Duester G, Plutzky J. Retinaldehyde represses adipogenesis and diet-induced obesity. Nat Med. 2007; 13:695-702.

14. Lin JJ, Shaw AT. Resisting resistance: targeted therapies in lung cancer. Trends Cancer. 2016; 2:350-364.

15. Giard DJ, Aaronson SA, Todaro GJ, Arnstein P, Kersey JH, Dosik H, Parks WP. In vitro cultivation of human tumors: establishment of cell lines derived from a series of solid tumors. J Natl Cancer Inst. 1973; 51:1417-1423.

16. Ge X, Chen Q, Wu YP, Zhang Y, Xia H, Yuan D, Chen Q, Leng W, Chen L, Tang Q, Pang X, Bi F. Induced IGF-1R activation contributes to gefitinib resistance following combined treatment with paclitaxel, cisplatin and gefitinib in A549 lung cancer cells. Oncol Rep. 2014; 32:1401-1408.

17. Moreb JS, Zucali JR, Ostmark B, Benson NA. Heterogeneity of aldehyde dehydrogenase expression in lung cancer cell lines is revealed by Aldefluor flow cytometry-based assay. Cytometry B Clin Cytom. 2007; 72:281-289.

18. Lidén M, Eriksson U. Understanding retinol metabolism: structure and function of retinol dehydrogenases. J Biol Chem. 2006; 281:13001-13013.
19. Mihai DM, Washington I. Vitamin A dimers trigger the protracted death of retinal pigment epithelium cells. Cell Death Dis. 2014; 5:e1348.

20. He M, Du W, Du Q, Zhang Y, Li B, Ke C, Ye Y, Du Q. Isolation of the retinal isomers from the isomerization of all-trans-retinal by flash countercurrent chromatography. J Chromatogr A. 2013; 1271:67-70.

21. Fishkin N, Yefidoff R, Gollipalli DR, Rando RR. On the mechanism of isomerization of all-trans-retinol esters to 11-cis-retinol in retinal pigment epithelial cells: 11-fluoroall-trans-retinol as substrate/inhibitor in the visual cycle. Bioorg Med Chem. 2005; 13:5189-5194.

22. Novitskiy G, Traore K, Wang L, Trush MA, Mezey E. Effects of ethanol and acetaldehyde on reactive oxygen species production in rat hepatic stellate cells. Alcohol Clin Exp Res. 2006; 30:1429-1435.

23. Arellanes-Robledo J, Reyes-Gordillo K, Shah R, Domínguez-Rosales JA, Hernández-Nazara ZH, Ramirez F, Rojkind M, Lakshman MR. Fibrogenic actions of acetaldehyde are $\beta$-catenin dependent but Wingless independent: a critical role of nucleoredoxin and reactive oxygen species in human hepatic stellate cells. Free Radic Biol Med. 2013; 65:1487-1496.

24. Yan T, Zhao Y, Zhang X. Acetaldehyde induces cytotoxicity of SH-SY5Y cells via inhibition of Akt activation and induction of oxidative stress. Oxid Med Cell Longev. 2016; 2016:4512309.

25. Sawada O, Perusek L, Kohno H, Howell S, Maeda A, Matsuyama S, Maeda T. All-trans-retinal induces Bax activation via DNA damage to mediate retinal cell apoptosis. Exp Eye Res. 2014; 123:27-36.

26. Chen Y, Okano K, Maeda T, Chauhan V, Golczak M, Maeda A, Palczewski K. Mechanism of all-trans-retinal toxicity with implications for stargardt disease and age-related macular degeneration. J Biol Chem. 2012; 287:5059-5069.

27. Mizuno T, Suzuki N, Makino H, Furui T, Morii E, Aoki H, Kunisada T, Yano M, Kuji S, Hirashima Y, Arakawa A, Nishio S, Ushijima K, et al. Cancer stem-like cells of ovarian clear cell carcinoma are enriched in the ALDHhigh population associated with an accelerated scavenging system in reactive oxygen species. Gynecol Oncol. 2015; 137:299-305.

28. Lee SA, Belyaeva OV, Popov IK, Kedishvili NY. Overproduction of bioactive retinoic acid in cells expressing disease-associated mutants of retinol dehydrogenase 12 . J Biol Chem. 2007; 282:35621-35628.

29. Zannoto-Filho A, Schröder R, Moreira JC. Xanthine oxidase-dependent ROS production mediates vitamin A pro-oxidant effects in cultured Sertoli cells. Free Radic Res. 2008; 42:593-601.

30. Paika J, Blaner WS, Swisshelm K. Cis-retinol dehydrogenase: 9-cis-retinol metabolism and its effect on 
proliferation of human MCF7 breast cancer cells. Exp Cell Res. 2005; 303:183-196.

31. Koppaka V, Thompson DC, Chen Y, Ellermann M, Nicolaou KC, Juvonen RO, Petersen D, Deitrich RA, Hurley TD, Vasiliou V. Aldehyde dehydrogenase inhibitors: a comprehensive review of the pharmacology, mechanism of action, substrate specificity, and clinical application. Pharmacol Rev. 2012; 64:520-539.

32. Morgan CA, Parajuli B, Buchman CD, Dria K, Hurley TD. Using N, N-diethylaminobenzaldehyde (DEAB) as a substrate and mechanism-based inhibitor for human ALDH isoenzymes. Chem Biol Interact. 2015; 234:18-28. 